Article

\title{
Investigation of Pinewood Nematodes in Pinus tabuliformis Carr. under Low-Temperature Conditions in Fushun, China
}

\author{
Long Pan ${ }^{1}$, Rong Cui ${ }^{1,2}$, Yongxia Li ${ }^{1,3, *}$, Yuqian Feng ${ }^{1}$ and Xingyao Zhang ${ }^{1,3}$ \\ 1 Research Institute of Forestry New Technology, Chinese Academy of Forestry, Beijing 100091, China; \\ Longpan@caf.ac.cn (L.P.); cuibaobei93@126.com (R.C.); Yuqianfeng@caf.ac.cn (Y.F.); xyzhang@caf.ac.cn (X.Z.) \\ 2 Research Centre of Sub-frigid zone Forestry, Chinese Academy of Forestry, Harbin 150080, China \\ 3 Co-Innovation Center for Sustainable Forestry in Southern China, Nanjing Forestry University, \\ Nanjing 210037, China \\ * Correspondence: xinjishusuoli@163.com
}

Received: 4 August 2020; Accepted: 9 September 2020; Published: 16 September 2020

check for updates

\begin{abstract}
In recent years, the pinewood nematode has continuously adapted to low-temperature environments and expanded from the South to the North of China. In December 2018, a large area of pinewood nematode was suspected to be harmful to Pinus tabuliformis under natural conditions in Fushun City, Liaoning Province. In order to clarify the low-temperature environment and population characteristics of pinewood nematodes in this new epidemic area, we analyzed the difference in temperature between the inside and outside of $P$. tabuliformis in low-temperature environments, conducted the morphological and molecular identification of pinewood nematodes in P. tabuliformis, summarized the distribution characteristics of the wintering of pinewood nematodes and explored the population structure of pinewood nematodes under different low-temperature conditions. The results indicated that the diurnal variation of temperature in dead P. tabuliformis was significantly less than the environment temperature. The lowest temperature in P. tabuliformis was $3.2^{\circ} \mathrm{C}$ higher than the lowest temperature in the environment in one day; the pathogen of a large area of dead P. tabuliformis in Fushun was pinewood nematode (Bursaphelenchus xylophilus); $84.9 \%$ of the average populations of pinewood nematodes were third-stage dispersal juveniles, which mainly gathered in $5 \mathrm{~cm}$ within the pupal chamber of Monochamus saltuarius Gebler. At $-40{ }^{\circ} \mathrm{C}$, most of the third-stage dispersal juveniles of pinewood nematode in dead pine can still survive. Our study laid a foundation for the understanding of the low-temperature adaptation mechanism of pinewood nematode and contributed to the monitoring of pine wilt disease in the mid-temperate zone.
\end{abstract}

Keywords: low temperature; Bursaphelenchus xylophilus; third-stage dispersal juveniles

\section{Introduction}

Pine wilt disease is a complex disease caused by Bursaphelenchus xylophilus (Steiner \& Buhrer). Nickel and the insects of Monochamus spp. are the main mediators. When the environment of pinewood nematodes changes unfavorably, pinewood nematodes will transform from propagative juveniles to dispersal juveniles. The third-stage dispersal juveniles are able to resist adverse environments [1]. These Monochamus saltuarius Gebler migrate to healthy pine trees to help the spread of pine wilt disease when seeking food from tender branches [2]. Natural susceptible hosts in the traditional distribution area of pine wilt disease in China include Pinus kesiya, Pinus massoniana, Pinus armandii and Pinus thunbergii [3]. In 2016, pinewood nematodes were found to be harmful to P. thunbergii in Dalian, Liaoning Province and Pinus koraiensis in Dandong City, Liaoning Province in 2017 [4]. In recent years, 
pinewood nematodes have continuously adapted to the low-temperature environment and expanded from the South to the North. In the process, they continuously adapt to new hosts.

Pinus tabuliformis has needle-like leaves, usually in pairs. It is an evergreen tree of the genus of pinus and has strong cold hardiness which allows it to endure temperatures as low as $-25^{\circ} \mathrm{C}$ [5]. P. tabuliformis is a pine native to China from the provinces of the Northeast, Mid-East and Northwest of China, such as Beijing, Liaoning, Jilin, Inner Mongolia and other provinces [6]. P. tabuliformis is well known for its economic value and has important ecological benefits such as climate regulation, water conservation, wind prevention and sand fixation, as well as the conservation of species diversity. P. tabuliformis is planted in large areas such as Beijing, Liaoning and Jilin in China. Large economic losses were felt when the trees were harmed by pine wilt disease.

The temperature in pines changes with the environment, but in a low-temperature environment, due to the accumulation of cold-resistant substances and the impact of physiological activity, pine temperature and environment temperature are different [7]. The annual average temperature of pinewood nematodes in China's traditional distribution area is above $10{ }^{\circ} \mathrm{C}$. Fushun City, Liaoning Province is located in the middle temperate zone influenced by China's monsoon season. The annual average temperature is $6.7^{\circ} \mathrm{C}$, and the minimum temperature in winter is around $-30^{\circ} \mathrm{C}[8]$. At the end of 2018, an epidemic situation suspected of pinewood nematode damage in P. tabuliformis was found in Fushun City, so it was vital to identify the pathogenic nematodes. Moreover, pinewood nematodes can live through the winter in pines; therefore, its community characteristics and the low-temperature conditions in the new epidemic area were poorly understood.

\section{Materials and Methods}

\subsection{Differences in Temperature between the Inside and Outside of P. tabuliformis}

In December 2018, 30 P. tabuliformis in the same sample plot were randomly selected and were divided into two levels according to the features of pine wilt disease, 15 in each level. A $6 \mathrm{~cm}$-deep hole was drilled at a height of $1.5 \mathrm{~m}$ of the pine trees with the use of a handheld electric drill (WORX, CHN, Beijing, China), and the temperature both inside or outside of the pine was simultaneously measured with a contact thermometer (TES, Inc., Massachusetts, USA,). Temperature data were recorded every $1 \mathrm{~h}$ from 6:00 to 18:00, so as to determine the variation of temperature in P. tabuliformis.

\subsection{Morphological Characteristics of Pinewood Nematode under Low Temperature}

In December 2018, 15 dead P. tabuliformis were randomly selected and the trunk wood discs were collected. The Baermann funnel method was used for nematode extraction. The collected P. tabuliformis discs were chopped into wooden pieces of about $1 \times 1 \times 0.5 \mathrm{~cm}$, placed in a funnel and the appropriate amount of distilled water was added in order to submerge the blocks. After standing at room temperature of $25^{\circ} \mathrm{C}$ for $24 \mathrm{~h}$, the isolated nematode solution was collected with a centrifuge tube. Then, the solution was centrifuged and $2-3 \mathrm{~mL}$ of the centrifuged liquid was taken from the bottom of the tube for microscopic examination. The pinewood nematode was picked out with a $10 \mu \mathrm{L}$ pipette and dripped onto the concave slide. The slide was placed over the flame of the alcohol lamp for 3-5 s. After the pinewood nematode was heat-killed, the nematode presented an open bracket shape. Then, a temporary slide of the pinewood nematode was made for observing and measuring the nematode morphology.

The morphological identification of pinewood nematodes was based on a previous study [9]. The third-stage dispersal juveniles of pinewood nematodes also had distinct characteristics such as obvious constriction, fine stylet, an ovoid-shaped oesophageal bulb and a large number of fat particles in the body. The tail of this pinewood nematode is finger-like [10]. 


\subsection{Molecular Identification of Pinewood Nematode}

A total of 50 nematodes were picked under a microscope with a $10 \mu \mathrm{L}$ pipette and the nematode DNA was extracted according to the procedures of DNA preparation kit AP-MN-MS-GDNA-50 purchased from Axygen, USA. The PCR reaction volume was $25 \mu \mathrm{L}$ in total, which included $12.5 \mu \mathrm{L}$ PCR Mix, $1 \mu \mathrm{L}$ of upstream and $1 \mu \mathrm{L}$ of downstream primers, $1 \mu \mathrm{L}$ DNA template and $9.5 \mu \mathrm{L}$ of $\mathrm{ddH}_{2} \mathrm{O}$ [11]. Upstream primer: 5'-CGT AAC AAG GTA GCT GTA-3, downstream primer: 5'-TTT CAC TCG CCG TTA CTA AGG-3. PCR procedure: $94{ }^{\circ} \mathrm{C}$, initiation $2 \mathrm{~min} ; 94{ }^{\circ} \mathrm{C}$ denaturation 1 min, $55^{\circ} \mathrm{C}$ annealing $1 \mathrm{~min}, 72{ }^{\circ} \mathrm{C}$ extension $1 \mathrm{~min}$, a total of 35 cycles; $72{ }^{\circ} \mathrm{C}$ the final extension for $5 \mathrm{~min}$ [12]. The PCR product was sent to the gene sequencing company and the amplified sequence was contrasted with the B. xylophilus in NCBI.

\subsection{Distribution Characteristics of Pinewood Nematode in P. tabuliformis}

In December 2018, three damaged P. tabuliformis were randomly harvested in the sample plots of pine wilt disease, and $5 \mathrm{~cm}$-thick wooden discs were collected at the bottom, middle, and top parts of the trunk. Then, we looked for a pupal chamber of Monochamus saltuarius Gebler in the collected wooden disc. Small pieces of wood of $1 \times 1 \times 1 \mathrm{~cm}$ were cut from the cross-section of the area of the disc, which were 1-10 cm away from the pupal chamber, and collected every other $1 \mathrm{~cm}$. Then, the wood blocks were soaked with distilled water in a culture dish for $24 \mathrm{~h}$. The number of the third-stage dispersal juveniles of pinewood nematodes was counted on an inverted microscope.

\subsection{Population Development Characteristics of B. xylophilus in P. tabuliformis}

The collected pinewood discs were cut into $5 \times 5 \times 5 \mathrm{~cm}$ blocks and placed in $-40{ }^{\circ} \mathrm{C},-20^{\circ} \mathrm{C}, 4^{\circ} \mathrm{C}$ and $25^{\circ} \mathrm{C}$ temperatures. Two days later, the collected wooden blocks were cut into $1 \times 1 \times 0.5 \mathrm{~cm}$ small pieces of wood. The blocks were placed into five $10 \mathrm{~cm}$ culture dishes according to the average mass of wood, and then soaked with distilled water for $24 \mathrm{~h}$. The average survival number of pinewood nematodes and the ratio of survival pinewood nematodes of different ages were counted under the microscope. The control group was composed of the wood discs of the same size and collected from the same plots, but without any temperature treatment, and the data of the control group were collected immediately once the nematodes were isolated. The nematodes isolated by the Baermann funnel method included a variety of saprophytic nematodes and entomopathogenic nematodes in addition to pinewood nematodes. The nematodes were isolated by the Baermann funnel method [13].

\section{Results}

\subsection{Inside and Outside Temperature Difference of P. tabuliformis}

According to the meteorological data on the day of measurement, the lowest temperature in Fushun appeared around 7:00, where a low-temperature inflection point was formed. The highest temperature appeared around 14:00, where a high-temperature inflection point was formed. It can be seen from Figure 1 that the variation trend of the temperature in the dead P. tabuliformis (initially rising then descending) was in accordance with the environment temperature. However, the temperature variation in dead P. tabuliformis was significantly lower than the environment temperature. The temperature change in healthy P. tabuliformis was smaller than that of the dead pine and remained more stable. The low-temperature inflection point of dead P. tabuliformis appeared about $1 \mathrm{~h}$ later than the environment temperature. The lowest temperature was $3.2^{\circ} \mathrm{C}$ higher than the lowest temperature of the environment, and the highest temperature was $2.1^{\circ} \mathrm{C}$ lower than the highest temperature of the environment. 


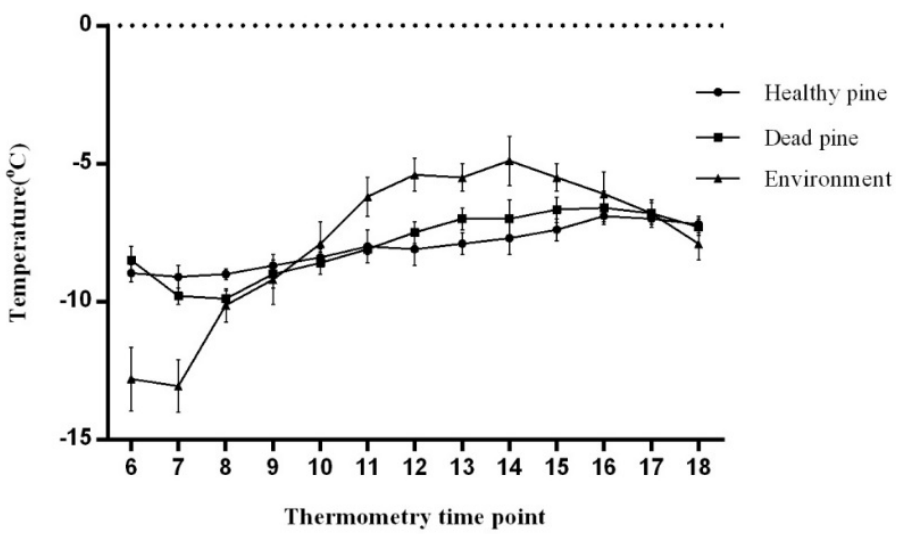

Figure 1. Variation of temperature inside and outside of $P$. tabuliformis over time.

\subsection{Morphological Characteristics of the Third-Stage Dispersal Juveniles of Pinewood Nematode in} P. tabuliformis

The nematode was isolated from the trunks of 15 dead P. tabuliformis. The tip of the head was obviously constricted, the stylet was slender and the median oesophageal bulb was ovoid in shape, which accounted for about two-thirds of the body width (Figure 2B). The body was full of fat granules except for the head (Figure 2A,C,D) and the tails were finger-like (Figure 2D). The above observations are consistent with the characteristics of the dispersal type pinewood nematode. The pinewood nematodes in Fushun P. tabuliformis were suspected to be third-stage dispersal juveniles, and their size is shown in Table 1.
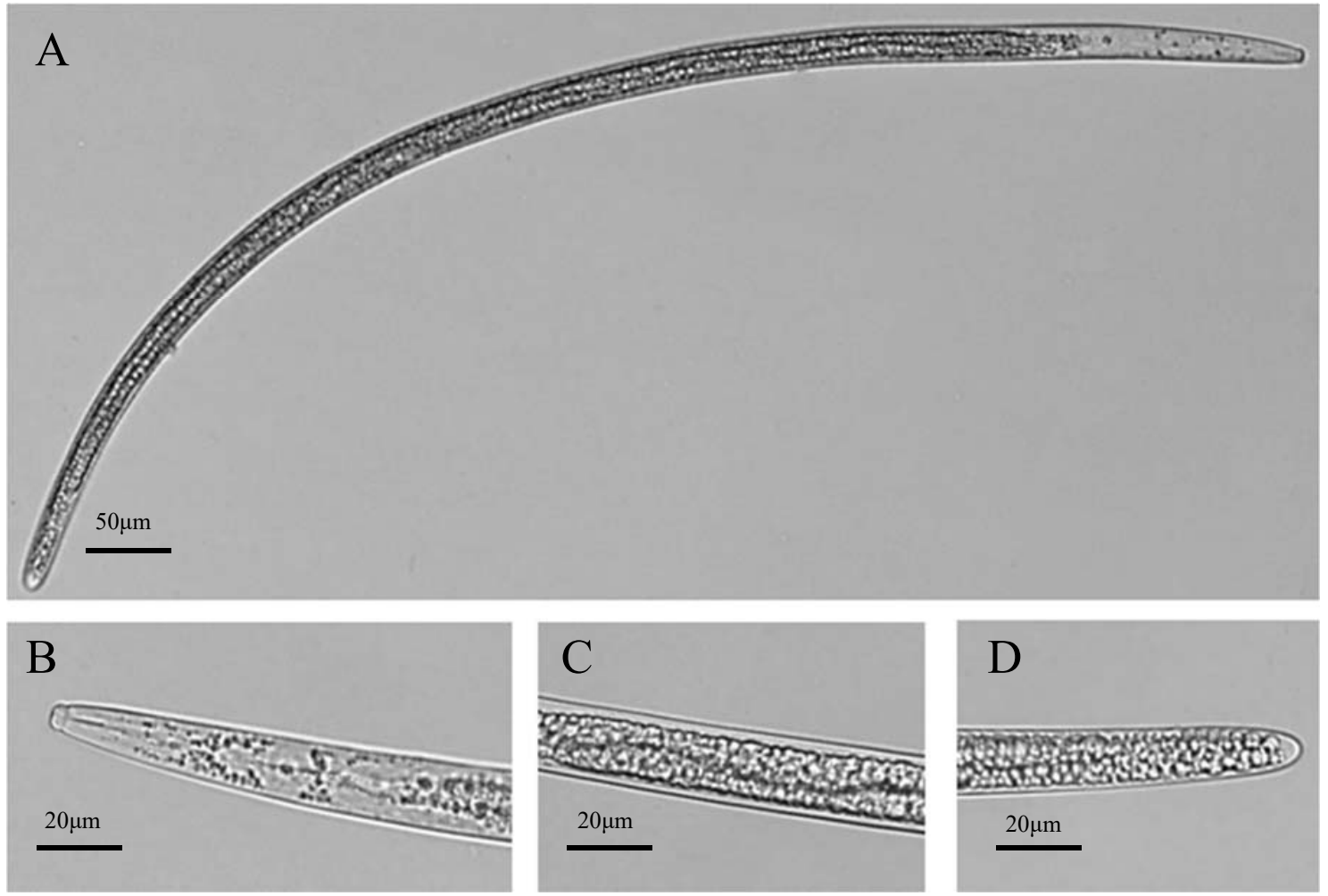

Figure 2. Morphological characteristics of the third-stage dispersal juveniles of pinewood nematode isolated from P. tabuliformis in Fushun. (A) Whole body of the third-stage dispersal juveniles of pinewood nematode; (B) head of the third-stage dispersal juveniles of pinewood nematode; (C) midbody of the third-stage dispersal juveniles of pinewood nematode; (D) tail of the third-stage dispersal juveniles of pinewood nematode. 
Table 1. Morphological measurements of third-stage dispersal juveniles of pinewood nematode isolated from P. tabuliformis in Fushun.

\begin{tabular}{cccccccc}
\hline PWN & $\mathbf{N}$ & L/ $/$ m & a & b & c & St $/ \boldsymbol{\mu m}$ & Tail $/ \mu \mathrm{m}$ \\
\hline PWN DL $_{3}$ & 30 & $601.05 \pm 23.18$ & $36.82 \pm 1.32$ & $9.35 \pm 0.11$ & $14.20 \pm 0.56$ & $8.66 \pm 0.18$ & $42.35 \pm 0.17$ \\
\hline
\end{tabular}

Note: PWN—pinewood nematode.N—the number of samples detected; L—body length $(\mu \mathrm{m})$; a—length/maximum body width; $\mathrm{b}$ - distance between the body/body front and the junction of esophagus and intestine; c-length/tail length; Tail—tail length $(\mu \mathrm{m})$; St—needle length $(\mu \mathrm{m})$; the second value is the averaged $+/-\mathrm{SE}$.

\subsection{Molecular Identification of Pinewood Nematode in P. tabuliformis}

PCR amplification and sequencing was conducted with the sequence-specific primers of ITS and the template of nematode DNA of P. tabuliformis in Fushun. The length of the fragment was $815 \mathrm{bp}$. The sequence was blasted with the sequences in NCBI, and the result indicated that its similarity and coverage were $100 \%$, and the E value was 0 when aligned with MN006173.1, KF025323.1 and EF446943.1 (Supplementary Materials). Integrating the results of morphological identification and the above molecular sequencing results determined that the nematodes in P. tabuliformis of Fushun City were pinewood nematodes.

\subsection{Distribution Characteristics of the Third-Stage Dispersal Juveniles of Pinewood Nematode in P. tabuliformis}

By statistically analyzing the third-stage dispersal juveniles of pinewood nematodes at different sites of the wood near the pupal chamber of Monochamus saltuarius Gebler, it was found that the amount of nematodes at different locations was significantly different. The number of nematodes dropped rapidly with a gradual increase in distance from the pupal chamber. An average of $75.7 \%$ of third-stage dispersal juveniles of pinewood nematodes accumulated within $5 \mathrm{~cm}$ of the pupal chamber of Monochamus saltuarius Gebler (Figure 3).

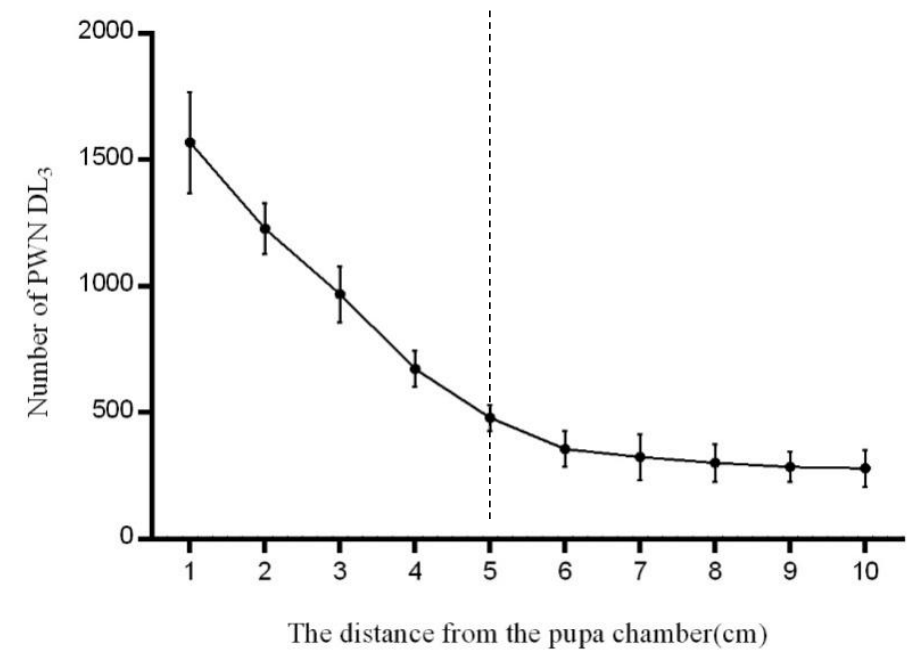

Figure 3. Number of third-stage dispersal juveniles of pinewood nematodes in blocks at different distances near the pupal chamber of Monochamus saltuarius Gebler. PWN DL 3 : third-stage dispersal juveniles of pinewood nematode.

\subsection{Population Development Characteristics of Third-Stage Dispersal Juveniles of Pinewood Nematodes at Different Temperatures}

From the CK control group in Figure 4, it can be determined that the nematodes isolated from P. tabuliformis in Fushun City in December included saprophytic nematodes, insect parasitic nematodes, and pinewood nematodes, of which the average proportion of pinewood nematodes was $71.5 \%$. Of these pinewood nematode populations, an average of $84.9 \%$ were third-stage dispersal juveniles and $10.9 \%$ adult. On average, more than $62 \%$ of the pinewood nematodes were diffuse third-stage dispersal juveniles at temperatures of $-40^{\circ} \mathrm{C},-20^{\circ} \mathrm{C}, 4^{\circ} \mathrm{C}$ and $25^{\circ} \mathrm{C}$. The proportion of third-inferiority types 
of pinewood nematodes in the pinewood segment gradually decreased with the gradual increase of temperature, whereas the adult and reproductive larvae of the pinewood nematode gradually increased. Treating the P. tabuliformis for 2 days at $-40{ }^{\circ} \mathrm{C}$ caused all of the adults and larvae of the pinewood nematode to die, whereas all of the remaining third-stage dispersal juveniles were able to survive in large numbers.

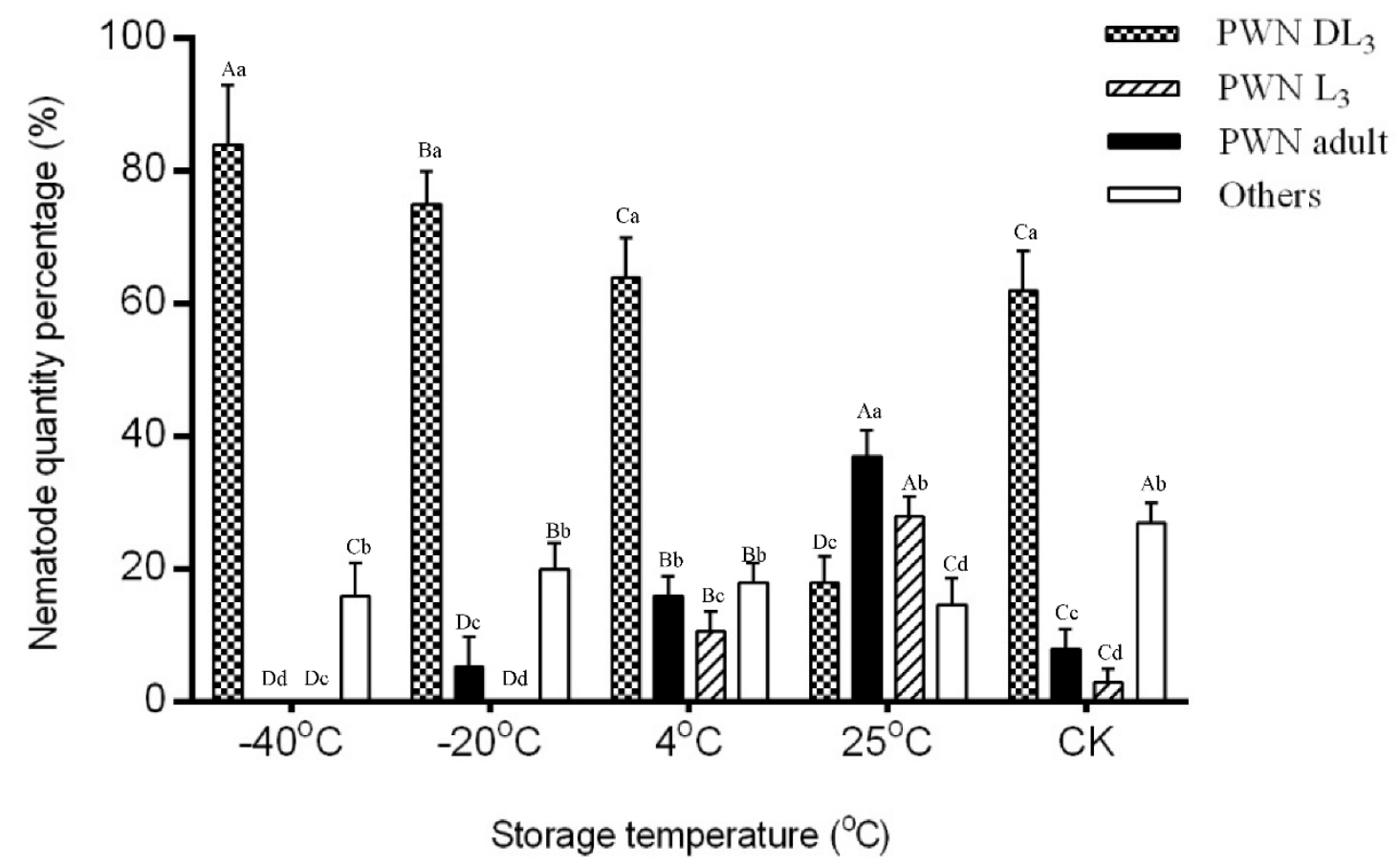

Figure 4. Proportion of larvae at different stages of development of pinewood nematode in P. tabuliformis under different temperatures. $\mathrm{PWN} \mathrm{DL}_{3}$ : third-stage dispersal juveniles of pinewood nematode; PWN L $\mathrm{L}_{3}$ : third-stage larvae of reproduction of pinewood nematode; PWN adult: adult pinewood nematode. Note: The first capital letter represents the same PWN growth stage at different temperatures in the amount of significant nematode differences between different letters. The second lowercase letter represents the significant difference of nematode content at different PWN growth stages at the same temperature, and the significant difference between different letters.

\section{Discussion}

Pinewood nematodes have spread rapidly throughout China since being introduced to Nanjing, China in 1982. The traditional distribution areas are in the monsoon-controlled warm temperate zone and subtropical regions, where the average temperature of the warm temperate zone is between 8 and $13{ }^{\circ} \mathrm{C}$ and the annual temperature in subtropical region is between 13 and $23^{\circ} \mathrm{C}$ on average. The average annual temperature of Fushun is $6.7^{\circ} \mathrm{C}$, and the lowest temperature in winter is below $-30^{\circ} \mathrm{C}$. The pinewood nematode belongs to the middle temperature zone, where the annual average temperature is between 2 and $8{ }^{\circ} \mathrm{C}$. The invasion of pinewood nematodes into Fushun indicates that these nematodes have entered the middle temperate zone and generated adaptations to the low-temperature environment in genetics and physiology. The ancestors of B. xylophilus originated in the colder eastern part of Eurasia genetically [12]. In Quebec, North America, pinewood nematodes differentiate as an independent species and are widely distributed across North America in average temperatures of $-20^{\circ} \mathrm{C}$ to $20^{\circ} \mathrm{C}$ [14]. Pinewood nematodes possess genes and epigenetic modifications that can deal with harsh environments such as low temperatures, and thus hold the ability to adapt to environmental stress rapidly. Physiologically, the contents of trehalose, glucose and glycerol in pinewood nematodes increased significantly in the low-temperature environment $[15,16]$. The variation trend of trehalose content showed a significant correlation with the survival rate of pinewood nematodes at low temperatures [17]. 
In field conditions, the day temperature in pine trees changes smoothly, and the lowest temperature is slightly higher than the environment, which is conducive to wintering pinewood nematodes. When the third-stage dispersal juveniles of pinewood nematodes were placed in distilled water and in $\mathrm{a}-20{ }^{\circ} \mathrm{C}$ environment for $1.5 \mathrm{~h}$, all of the pinewood nematodes died. However, a large number of live pinewood nematodes could be isolated from the P. tabuliformis and treated with the same conditions. The results above also indicate that the pine tree itself may be able to help the pinewood nematode resist low temperatures in order to help it to survive the winter [18]. However, the specific regulatory mechanism remains to be further elucidated.

No previous study of pinewood nematodes that endangered P. tabuliformis under natural conditions has been reported. We reported for the first time that pinewood nematodes endanger the occurrence of P. tabuliformis in the monsoon-controlled middle temperate zone of China, which signifies that the pinewood nematode has begun to harm new hosts. Previous studies have shown that 106 species can be infected by pinewood nematodes, most of which are plants in Pinus, but also include plants in Abies, Larix and so on [19,20], among which P. tabuliformis, Pinus koraiensis, Pinus sylvestris, Larix gmelinii and Abies were widely affected in the middle temperate zone. There are 13 species of vector insects of pinewood nematode, all of which are of the Monochamus spp. [4], in which Monochamus saltuarius Geblersaltuarius and Monochamus saltuarius Geblersutor are distributed in the middle temperate zone, which overlaps with the potential host distribution area of the pinewood nematode. The outbreaks of pinewood nematode disease were detected in Dalian in 2016, Dandong in 2017 and Fushun in 2018, which suggest that the pinewood nematode disease is spreading rapidly. It is vital to monitor pinewood nematode vector insects in the new epidemic area and predict the potential host and occurrence of pine wilt disease in nonepidemic areas of the middle temperate zone, as well as strengthen the monitoring and early warning signs of pine wilt disease.

Supplementary Materials: The following are available online at http://www.mdpi.com/1999-4907/11/9/993/s1.

Author Contributions: L.P. wrote articles and experiment; R.C. did experiment. Y.L. guided article writing. Y.F. collected the samples. X.Z. guided article writing. All authors have read and agreed to the published version of the manuscript.

Funding: This study was supported by Chinese Academy of Forestry Program (CAFYBB2019SY302).

Conflicts of Interest: The authors declare no conflict of interest.

\section{References}

1. Zhao, L.; Zhang, X.; Wei, Y.; Zhou, J.; Zhang, W.; Qin, P.; Chinta, S.; Kong, X.; Liu, Y.; Yu, H.; et al. Ascarosides coordinate the dispersal of a plant-parasitic nematode with the metamorphosis of its vector beetle. Nat. Commun. 2016, 7, 1-8. [CrossRef] [PubMed]

2. Zhao, L.; Zhang, S.; Wei, W.; Hao, H.; Zhang, B.; Butcher, R.A.; Sun, J. Chemical signals synchronize the life cycles of a plant-parasitic nematode and its vector beetle. Curr. Biol. 2013, 23, 2038-2043. [CrossRef] [PubMed]

3. Futai, K. Pinewood Nematode, Bursaphelenchus xylophilus. Annu. Rev. Phytopathol. 2013, 51, 61-83. [CrossRef] [PubMed]

4. Pan, L.; Li, Y.X.; Liu, Z.K.; Meng, F.L.; Chen, J.; Zhang, X.Y. Investigation and identification of pinewood nematode disease in Pinus koraiensis in Fengcheng, Liaoning province. For. Pest Dis. 2019, 38, 5-8.

5. Zhang, A.O.; Cui, Z.H.; Yu, J.L.; Hu, Z.L.; Ding, R.; Ren, M.; Zhang, L.J. Dissipation of excess excitation energy of the needle leaves in Pinus, trees during cold winters. Int. J. Biometeorol. 2016, 60, 1-8. [CrossRef] [PubMed]

6. Zhang, L.; Liu, S.; Sun, P.; Wang, T. Partitioning and mapping the sources of variations in the ensemble forecasting of species distribution under climate change: A case study of Pinus tabulaeformis. Acta Ecol. Sin. 2011, 31, 5749-5761.

7. Merry, R.; Jerrard, J.; Frebault, J.; Verhoeven, A. A comparison of pine and spruce in recovery from winter stress; changes in recovery kinetics, and the abundance and phosphorylation status of photosynthetic proteins during winter. Tree Physiol. 2017, 37, 1239-1250. [CrossRef] [PubMed] 
8. Liu, M.; Fu, X.; Yang, L.; Bing-kun, L.I.; Lei, M.I.; Zhang, H.; Jian, M.A.; Zhang, B. Characteristics of main climatic elements from 1960 to 2013 in Fushun region. J. Meteorol. Environ. 2015, 31, 140-146.

9. Yamanobe, T. Relationships between morphological traits and resistance to pinewood nematode in two Japanese pines. Eur. J. Plant Pathol. 2009, 124, 543-552. [CrossRef]

10. David, G.; Giffard, B.; Piou, D.; Jactel, H. Dispersal capacity of Monochamus saltuarius Gebler galloprovincialis, the European vector of the pinewood nematode, on flight mills. J. Appl. Entomol. 2014, 138, 566-576. [CrossRef]

11. Takeuchi, Y.; Kanzaki, N.; Futai, K. A nested PCR-based method for detecting the pinewood nematode, Bursaphelenchus xylophilus, from pinewood. Nematology 2005, 7, 775-782.

12. Kanzaki, N.; Futai, K. A PCR primer set for determination of phylogenetic relationships of Bursaphelenchus species within the xylophilus group. Nematology 2002, 4, 35-41. [CrossRef]

13. Jikumaru, S.; Togashi, K. Boarding abilities of Bursaphelenchus mucronatus and B. xylophilus (Nematoda: Aphelenchoididae) on Monochamus saltuarius Gebler alternatus (Coleoptera: Cerambycidae). Nematology 2003, 5, 843-849.

14. Pereira, F.; Moreira, C.; Fonseca, L.; van Asch, B.; Mota, M.; Abrantes, I.; Amorim, A. New Insights into the Phylogeny and Worldwide Dispersion of Two Closely Related Nematode Species, Bursaphelenchus xylophilus and Bursaphelenchus mucronatus. PLoS ONE 2013, 8, e56288. [CrossRef] [PubMed]

15. Klosin, A.; Casas, E.; Hidalgo-Carcedo, C.; Vavouri, T.; Lehner, B. Transgenerational transmission of environmental information in C. elegans. Science 2017, 356, 320-323. [PubMed]

16. Hourize'Evi, L.; Korem, Y.; Sheftel, H.; Faigenbloom, L.; Toker, I.A.; Dagan, Y.; Awad, L.; Degani, L.; Alon, U.; Rechavi, O. A Tunable Mechanism Determines the Duration of the Transgenerational Small RNA Inheritance in C. elegans. Cell 2016, 165, 88-99. [CrossRef] [PubMed]

17. Huang, R.; Gao, R.; Shi, J.; Song, D.; Li, Z. Cold Tolerance of Bursaphelenchus xylophilus in the Three Gorges Region of Hubei Province. J. Northeast For. Univ. 2014, 11, 031.

18. Lamitina, T.; Huang, C.G.; Strange, K. Genome-wide RNAi screening identifies protein damage as a regulator of osmoprotective gene expression. Proc. Natl. Acad. Sci. USA 2006, 103, 12173-12178. [CrossRef] [PubMed]

19. Jones, J.T.; Moens, M.; Mota, M.; Li, H.; Kikuchi, T. Bursaphelenchus xylophilus: Opportunities in comparative genomics and molecular host-parasite interactions. Mol. Plant Pathol. 2008, 9, 357-368. [CrossRef] [PubMed]

20. Zhang, J.J.; Zhang, R.Z.; Chen, J.Y. Species and their dispersal ability of Monochamus saltuarius Gebler as vectors to transmit Bursaphelenchus Xylophilus. J. Zhejiang For. Coll. 2000, 24, 350-356. 